\title{
How to unmask masked hypertension: the role of office aortic blood pressure
}

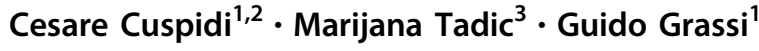

Received: 26 September 2020 / Revised: 1 October 2020 / Accepted: 1 October 2020 / Published online: 5 November 2020

(C) The Japanese Society of Hypertension 2020

The combination of office and out-of-office blood pressure (BP) measurements is increasingly used in current practice and clinical research, as this approach provides a more comprehensive assessment of cardiovascular risk related to hypertension than BP measured in the medical setting. Combining office and out-of-office (i.e., home and ambulatory) BP measurements, four different BP patterns may be identified, namely, normotension (normal office and out-ofoffice BP), sustained hypertension (elevated in-office and outof-office BP), white coat hypertension (elevated office and normal out-of-office BP), and the inverse phenomenon commonly defined as masked hypertension (MH) (normal office and elevated out-of-office BP). The four BP phenotypes differ widely from each other in their demographic/clinical correlates, conventional risk factors including hypertensionmediated organ damage (OD) and, more importantly, in their association with nonfatal and fatal cardiovascular events and all-cause mortality.

In particular, growing attention has been given to $\mathrm{MH}$, the pattern characterized by the classification of a normal BP status by measurements taken in a medical environment not being confirmed by ambulatory and/or home BP monitoring. It is also useful to point out that the definition of MH should be strictly reserved for untreated individuals because as treated individuals have normal BP values when measured in a medical setting, they are classified as normotensive in all respects [1]. Treated hypertensive patients presenting normal office BP and elevated ambulatory or home values should not be classified as MH but should rather be more appropriately

Cesare Cuspidi

cesare.cuspidi@unimib.it

1 Department of Medicine and Surgery, University of Milano-Bicocca, Milano, Italy

2 Istituto Auxologico Italiano, Milano, Italy

3 Department of Cardiology, University Hospital "Dr. Dragisa Misovic-Dedinje", Belgrade, Serbia classified as having masked uncontrolled hypertension (MUH). This differentiation is not merely formal since these two conditions have different clinical, prognostic, and therapeutic correlates. Several lines of evidence support the view that BP measured outside the medical setting is more closely associated with OD (an intermediate step in the continuum linking traditional risk factors to overt cardiovascular disease) than traditional measurements in the physician's office. In the last two decades, the association of $\mathrm{MH}$ with left ventricular hypertrophy (LVH), the cardinal biomarker of subclinical OD, has been the object of cross-sectional and longitudinal studies and related meta-analyses.

In the Pressioni Arteriose Monitorate e Loro Associazioni (PAMELA) study, a prospective population-based observational study, an echocardiogram and office and ambulatory BP were simultaneously measured at baseline and after a 10-year follow-up, we investigated the value of $\mathrm{MH}$ as a predictor of new-onset LVH. Individuals fulfilling the criteria for $\mathrm{MH}$ at entry showed a much greater incidence of new-onset LVH over a 10-year follow-up than individuals with normal office and ambulatory BP values (36\% vs. 17\%) [2]. Of note, the risk of developing this adverse cardiac phenotype in MH subjects was still more than double that in true normotensive subjects after adjustment for major confounders and was only slightly lower than that observed in individuals with sustained hypertension. In a recent cross-sectional investigation of the PAMELA population, we showed that the extent of LV involvement, as assessed by a prognostically validated marker, the LV mass index, was independent of circumstances in which out-of-office BP elevation had been documented (i.e., isolated home BP, isolated ambulatory BP or both) [3]. This suggests that even a partial elevation in BP can play a key role in the pathogenesis of cardiac OD, and consequently, the complementary use of the two methods is recommended to identify a larger number of $\mathrm{MH}$ subjects with high cardiovascular risk.

In parallel with the available data on OD, a large body of evidence supports the notion that the risk of cardiovascular 
events is substantially greater in individuals with $\mathrm{MH}$ than in their counterparts with normal in- and out-of-office BP and close to or even higher than observed in those with sustained hypertension. The pioneering study that first provided information on the adverse prognostic significance of $\mathrm{MH}$ was carried out by Bjorklund et al. in a sample of 570 untreated elderly men [4]. During a follow-up of 8.4 years, individuals with $\mathrm{MH}$, detected by ambulatory $\mathrm{BP}$ monitoring, displayed a similar incidence of coronary events, stroke, and symptomatic peripheral vascular disease as those with sustained hypertension, with a relative risk approximately threefold higher than that observed in true normotensives.

Using ambulatory BP, MH and MUH can be defined as daytime and/or nighttime and/or 24-h BP above certain thresholds. The prognostic value of MUH defined by different ambulatory criteria was recently addressed by Coccina et al. in 738 treated hypertensive patients with normal clinical BP over 10 years of follow-up. Compared to controlled in- and out-of-office hypertension, the increased cardiovascular risk was lowest in individuals with nighttime MUH and highest in individuals with daytime $\mathrm{MUH}$, regardless of nighttime $\mathrm{BP}$ and daytime + nighttime MUH [5].

Altogether, these findings show that $\mathrm{MH}$ (and MUH) conveys a risk of subclinical cardiac and extracardiac OD and overt cardiovascular disease close to that associated with sustained hypertension. In view of the fact that a systematic search for both BP phenotypes (and particularly for $\mathrm{MH}$ ) at the community level is unfeasible, a more targeted screening aimed at subjects with a high pretest probability seems to be a viable alternative. Although the evidence in the literature is not entirely consistent, the factors playing a pivotal role in the elevation of out-ofoffice pressure in clinically normotensive subjects include high-normal office BP, obesity, smoking, excessive alcohol consumption, sleep apnea syndrome, age, and job stress. In the context of the scientific effort aimed at identifying BP elevation outside the medical setting in apparently normotensive individuals, the study by Gkaliagkousi et al. [6], published in this issue of the Journal, adds a new piece of information. Starting from the premise that compared to conventional clinic BP, aortic BP (central BP), derived from peripheral BP measurements using dedicated algorithms, may have incremental prognostic value in predicting subclinical OD and cardiovascular events [7-9], the authors investigated the impact of office aortic systolic BP (aSBP) measurements in detecting $\mathrm{MH}$ and $\mathrm{MUH}$ in two different cohorts (A and B). Cohort A consisted of 391 never-treated patients with essential hypertension (mean age 44 years, $58 \%$ men), whereas cohort B comprised 956 untreated and treated hypertensives (mean age 55 years, $52 \%$ men). In both cohorts, a single-visit measurement of both office brachial (b) (oscillometric device) and aortic BP (applanation tonometry on the radial artery with a Sphygmocor device) was performed. In patients belonging to cohort A, out-of-office BP was exclusively assessed by 24-h ambulatory BP monitoring, while the assessment of out-of-office BP values in cohort B was based on either 24-ambulatory BP or 7-day home BP monitoring.

In both cohorts, participants were classified into four office SBP patterns based on both bSBP and aSBP: type I: normal office bSBP $(<140 \mathrm{mmHg})$ and aSBP $(<130 \mathrm{mmHg}$, outcome-based threshold); type II: high office bSBP and normal office aSBP; type III: normal office bSBP and high office aSBP (isolated high office aSBP); and type IV: high office bSBP and aSBP. Of note, elevated aSBP was also classified according to the age- and sex-adjusted 90th percentile of the normal distribution of aSBP derived in a large healthy population.

Out-of-office hypertension was defined as daytime systolic $\mathrm{BP} \geq 130 \mathrm{mmHg}$ and/or diastolic $\mathrm{BP} \geq 80 \mathrm{mmHg}$ or home systolic $\mathrm{BP} \geq 135 \mathrm{mmHg}$ and/or diastolic $\mathrm{BP} \geq 85 \mathrm{mmHg}$, according to current European guidelines. The overall prevalence of $\mathrm{MH}$ in cohort A was 13\% (52 out of 391 patients), and 10 patients met the criteria for a type III pattern (isolated office aSBP equal to or higher than $130 \mathrm{mmHg}$ ). In this subgroup, the prevalence of $\mathrm{MH}$ was much higher (50\%) than that observed in the whole cohort. In cohort B, untreated and treated patients with normal office and elevated out-of-office BP (i.e., $\mathrm{MH}$ and MUH) represented $20 \%$ of the entire sample ( $n=956$ ). Among the 73 patients with "isolated high office aSBP" (i.e., type III pattern), MH/MUH could be identified in $\sim 57 \%$ of the cases. Interestingly, the type III pattern based on the age- and sex-adjusted 90th percentile of the normal distribution of aSBP was identified at a frequency 3.6 and 2.0 times higher than that reported with the threshold of $130 \mathrm{mmHg}$. Even using this diagnostic criterion, which identified a much larger number of subjects with elevated central BP, the prevalence of patients with $\mathrm{MH} / \mathrm{MUH}$ in the type 3 pattern ranged from $43 \%$ (cohort A) to $52 \%$ (cohort B). The consistency of these findings suggests that central blood pressure measurement may actually help unmask $\mathrm{MH}$ by strengthening the likelihood of identifying these patients based only on clinical criteria. Therefore, the study by Gkaliagkousi et al. [6] appears particularly worthy of praise as they have revealed a new perspective for a more precise identification of two dangerous $\mathrm{BP}$ phenotypes, $\mathrm{MH}$ and MUH. Finally, a few limitations of the study need to be mentioned.

First, a warning against limiting the definition of normal ambulatory BP monitoring to the daytime, as done in the present report, comes from a growing body of evidence because this approach is likely to prevent the detection of nighttime BP elevation in a large number of individuals. In fact, isolated nocturnal hypertension in the MH setting has been reported to be far from rare. Second, the classification 
of $\mathrm{MH}$ and MUH was based on a single set of office BP and ambulatory BP monitoring recordings. It should be noted that $\mathrm{MH}$ and MUH do not actually represent stable clinical traits. In the European Lacidipine Study on Atherosclerosis, it was shown that only $\sim 40 \%$ of patients exhibiting MUH in a first set of office and ambulatory BP measurements remained in the same condition at the second set of measurements [10].

\section{Compliance with ethical standards}

Conflict of interest The authors declare that they have no conflict of interest.

Publisher's note Springer Nature remains neutral with regard to jurisdictional claims in published maps and institutional affiliations.

\section{References}

1. Banegas JR, Ruilope LM, Williams B. White-coat uncontrolled hypertension, masked uncontrolled hypertension, and true uncontrolled hypertension, phonetic and mnemonic terms for treated hypertension phenotypes. J Hypertens. 2018;36:446-7.

2. Cuspidi C, Facchetti R, Quarti-Trevano F, Sala C, Tadic M, Grassi $\mathrm{G}$, et al. Incident left ventricular hypertrophy in masked hypertension. Hypertension. 2019;74:56-62.
3. Cuspidi C, Facchetti R, Quarti-Trevano F, Dell'Oro R, Tadic M, Grassi $\mathrm{G}$, et al. Left ventricular hypertrophy in isolated and dual masked hypertension. J Clin Hypertens. 2020;22:673-7.

4. Bjorklund K, Lind L, Zethelius B, Andren B, Lithell H. Isolated ambulatory hypertension predicts cardiovascular morbidity in elderly men. Circulation. 2003;107:1297-302.

5. Coccina F, Pierdomenico AM, Cuccurullo C, Pizzicannella J, Madonna R, Trubiani O, et al. Prognostic value of masked uncontrolled hypertension defined by different ambulatory blood pressure criteria. Am J Hypertens. 2020;33:726-33.

6. Gkaliagkousi E, Protogerou AD, Argyris AA, Koletsos N, Triantafyllou A, Anyfanti P, et al. Contribution of single office aortic systolic blood pressure measurements to the detection of masked hypertension: data from two separate cohorts. Hypertens Res. 2020. https://doi.org/10.1038/s41440-020-00550-9

7. Protogerou AD, Aissopou EK, Argyris A, Nasothimiou EG, Konstantonis GD, Karamanou M, et al. Phenotypes of office systolic blood pressure according to both brachial and aortic measurements: frequencies and associations with carotid hypertrophy in 1861 adults. J Hypertens. 2016;34:1325-30.

8. Ntineri A, Kollias A, Bountzona I, Servos G, Moyssakis I, Destounis A, et al. Twenty-four-hour ambulatory central blood pressure in adolescents and young adults: association with peripheral blood pressure and preclinical organ damage. J Hypertens. 2020;38:1980-8.

9. Vlachopoulos C, Aznaouridis K, O'Rourke MF, Safar ME, Baou $\mathrm{K}$, Stefanadis C. Prediction of cardiovascular events and all-cause mortality with central haemodynamics: a systematic review and metaanalysis. Eur Heart J. 2010;31:1865-71.

10. Mancia G, Facchetti R, Cuspidi C, Bombelli M, Corrao G, Grassi G. Limited reproducibility of MUCH and WUCH: evidence from the ELSA study. Eur Heart J. 2020;41:1565-1571. 